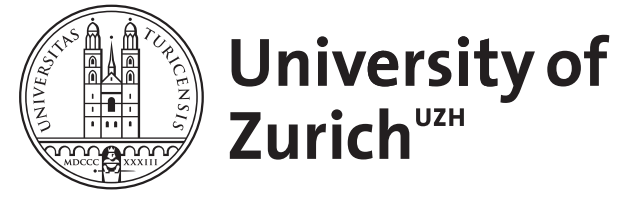

\title{
Das Kopfkissenbuch des Alten vom Berge
}

Schnyder, Mireille

DOI: https://doi.org/10.1515/9783110268799.203

Posted at the Zurich Open Repository and Archive, University of Zurich

ZORA URL: https://doi.org/10.5167/uzh-60994

Book Section

Published Version

Originally published at:

Schnyder, Mireille (2011). Das Kopfkissenbuch des Alten vom Berge. In: Gardt, Andreas; Schnyder, Mireille; Wolf, Jürgen. Buchkultur und Wissensvermittlung im Mittelalter und Früher Neuzeit. Berlin: De Gryter, 202-214.

DOI: https://doi.org/10.1515/9783110268799.203 


\section{Das Kopfkissenbuch des Alten vom Berge}

Der „Alte vom Berge“, wie der Führer des syrischen Zweigs der Assassinen in den Berichten der Kreuzfahrer genannt wird, war wie kaum eine zweite Figur dieser Zeit Objekt von Projektionen und Imaginationen. ${ }^{1}$ Die spärlichen Kenntnisse, die man von der Lehre und der Geschichte der nizāritischen 'Ismā'îliten hatte, ergänzt durch Berichte von Mordangriffen auf muslimische und christliche Herrscher, aber auch die konkreten Beziehungen zu dieser schwer einzuschätzenden Macht, führten zu einer Fülle von Gerüchten, Geschichten, Bildern und Ängsten. ${ }^{2}$ In den Berichten über die Lehre der Nizāriten, in der Regel aus der Feder ihrer Feinde, wurde das Bild einer häretischen religiösen Gruppierung gezeichnet, deren Adepten in blindem Gehorsam ihrem Meister gegenüber zu machtpolitisch motivierten Morden ausgebildet wurden, so dass durch sie Angst und Tod in die Herrschaftssysteme gebracht wurde. Dass die Anhänger der Lehre durch Drogen abhängig gemacht wurden, wurde zur Erklärung für den als „Haschischesser“ übersetzten Namen (arab.: Haššāšūn), ${ }^{3}$ und der paradiesische Garten des Alten vom Berge, der den Anhängern einen Vorgeschmack des Paradieses geben sollte, in das sie nach ihren selbstmörderischen Anschlägen einziehen würden, findet sich schon bald in fast jedem Reisebericht. Dabei war die Beschreibung jeweils gezeichnet durch eine Ambivalenz der Bewunderung

1 Die in Iran Ende des 11. Jh.s von Hasan-i Șabbāḥ gegründete 'ismā‘īlitische Sekte der Assassinen war durch ihre esoterische Glaubensgemeinschaft, ihre geheime Identität und ihre politisch eingreifende religiöse Präsenz ideales Objekte für Projektionen verschiedenster Art. Die sogenannte ,Neue Lehre‘ des Hasan-i Sabbāḥ grenzte sich nach dem Tod des fậtimidischen Kaliphen al-Mustanșir, der als Führer der 'Ismā‘̄iliten galt, von dessen Nachfolger in Ägypten ab und postulierte als rechtmäßigen Nachfolger seinen ältesten Sohn Nizār. Dieser war mit seinen Söhnen in Alexandrien zwar ermordet worden, doch installierte sich der Glaube, dass ein Enkel von ihm nach Alamūt gerettet werden konnte und sich die Linie so über die persischen 'Ismā'îliten fortsetzte. Unter Sinān ibn Salmān ibn Muhammad (Rašīd ad-Dīn), wurde im 12. Jh. in Syrien eine von dem persischen Zentrum in Alamūt weitgehend unabhängige Macht aufgebaut, die sich nach Sināns Tod wieder enger an Alamūt anschloss. Vgl. BERNARD LEWIS: The Assassins. A Radical Sect in Islam, London 1985, v.a. S. 97-124 (zu dem syrischen Zweig); FARHAD DAFTARY: The Ismāìlīs. Their history and doctrines, Cambridge, New York, Melbourne 1990, v.a. S. 396-403, 419-421 u.ö.

2 Zu den Projektionen vgl. FARHAD DAFTARY: The Assassin Legends. Myths of the Isma ilis, London, New York 1994. Auf Rašīd ad-Dīn Sinān geht die Bezeichnung, der Alte vom Berge' für die Syrischen Assassinenführer zurück. Vgl. dazu DAFTARY, S. $67 \mathrm{f}$.

3 Zur problematischen Etymologisierung und Erklärung des Namens vgl. DAFTARY (Anm. 2), S. 121-125. 
der in diesem Garten wirkenden technischen Einrichtungen und der Abwertung des darin installierten falschen Zaubers sowie magisch-teuflischer Machenschaften.

Selbst da, wo mit einem gewissen Interesse an Herrschaftsstruktur und Gedankengebäude der Nizāriten etwas weniger stark fabuliert wurde, wie bei Wilhelm von Tyrus oder - auf dessen Bericht fußend - auch bei Jacques de Vitry in seiner Historia orientalis ${ }^{4}$ beschränkt sich die Kenntnis auf ein paar wenige Punkte: die Gehorsamspflicht, die Idee, dass die Mörder als Märtyrer in das Paradies eingehen, die Erziehung der Adepten durch den Meister, die Bildung. Die Abtrünnigkeit der Assassinen vom sunnitischen Glauben wird zum Teil als Annäherung an das Christentum gesehen, bis dahin, dass die Konversion nur an der Einzeltat eines Christen gescheitert sein soll, der den Gesandten der Assassinen ermordete. ${ }^{5}$ Auch das sind Projektionen der Hoffnung, die sich nicht nur bei den Christen fanden, sondern auch bei muslimischen (persischen) Chronisten, wo die Geschichte jedoch auf eine Rückkehr in die Orthodoxie abzielte. ${ }^{6}$

Auf diesem Hintergrund ist es doch erstaunlich, oder zumindest auffallend, wenn in einem Bericht Ende des 13. Jh.s, im Rekurs auf einen Augenzeugen, ein fast schon intimer Blick auf das Buch neben dem Kopfkissen des Alten vom Berge geworfen wird. Die Textstelle findet sich in dem Livre des saintes paroles et des bons faiz nostre saint Roy Looÿs, das der Kreuzzugsteilnehmer und dann Vertraute des Königs Jean de Joinville (1225-1317) zwischen 1305 und 1309 verfasste. $^{7}$ Detailreich und anekdotenhaft ist in diesem Buch über das Leben des Königs auch der Kreuzzug (1248-1254) von Ludwig IX, dem Heiligen, dokumentiert ( $\S 106-654)$. Joinville, als Augenzeuge der Geschehnisse im Orient, gilt als präziser Beobachter, vertrauenswürdiger Zeuge und authentischer Berichterstatter. ${ }^{8}$ Wobei neuere Forschung deutlich macht, wie stark die-

4 Jacques de Vitry: Historia orientalis/Histoire orientale. Introduction, édition critique et traduction par JEAN DONNADIEU, Turnhout 2008, Kap. 14, S. 152-159.

5 Die Verhandlungen von Sinān mit dem König Amalric I wurden als Wille zur Konversion verstanden. So u.a. bei Wilhelm von Tyrus und Jacques de Vitry (Anm. 4), Kap. 14, 49-87. Vgl. Daftary (Anm. 2), S. 68, S. 71, S. 78f. Vgl. auch Alberich von Troisfontaines, der die „Nossorite“, wie sie bei ihm heißen, als Christen behandelt. Dazu: ANNA-DOROTHEE V. DEN BRINCKEN: „Die Nationes Christianorum Orientalium” im Verständnis der lateinischen Historiographie von der Mitte des 12. bis in die zweite Hälfte des 14. Jahrhunderts, Köln, Wien 1973, S. 359.

6 Dazu: Bernard Lewis: The Ismā'īlites and the Assassins. In: A History of the Crusades. Hrsg. von Kenneth M. Setton, Bd. 1, hrsg. von Marshall W. Baldwin, Madison, London ${ }^{2} 2006$, S. 99-134.

7 Joinville: Vie de saint Louis. Texte établi, traduit, présenté et annoté avec variantes par JACQUES MONFRIN, Paris 1995. Zur schwierigen und nicht eindeutig diskutierten Datierungsfrage vgl. ebd. S. LXVI-LXXVI; CAROLINE SMITH: Crusading in the age of Joinville, Aldershot 2006, S. 48-58; BRIGITTE STARK: ,La Vie de saint Louis' von Jean de Joinville. In: 1308. Eine Topographie historischer Gleichzeitigkeit. Hrsg. von A. SPEER/ D. WIRMER, Berlin 2010, S. 237-266, hier: S. 245f.

8 Vgl. MONFRIN (Anm. 7), S. I-II. 
ser , authentische' Bericht durch narrative Muster und Quellentexte geprägt ist, so dass er sich in ein Textnetz zeitgenössischer Literatur einfügt, von Enzyklopädik, chanson de geste bis Exempelliteratur. ${ }^{9}$

In Joinvilles Berichten vom Kreuzzug findet sich auch die Darstellung der Auseinandersetzung Ludwigs mit dem Alten vom Berge ( $\S ~ 451-462) .{ }^{10}$ Darin werden nicht nur die komplexen Machtverhältnisse in Szene gesetzt, sondern wird auch die Unsicherheit den unbekannten Glaubensinhalten gegenüber deutlich ( $\S 458-462)$. Dabei geht es Joinville in seiner Darstellung darum, einerseits die Macht des Königs auszustellen, anderseits Wissen über die unbekannte und geheimnisvolle Macht der Assassinen zu vermitteln. Das Kopfkissenbuch des Alten vom Berge dient ihm dabei als Schnittpunkt und Fokus verschiedener Projektionen, die sich auf Wissens- und Glaubenstraditionen gründen und wird so zum beglaubigenden Spiegel von eigener Wissenstradition. Wenn im Folgenden die Darstellung dieser Auseinandersetzung eng am Text aufgezeigt wird, geht es darum, nicht einfach die historische Begegnung zu erläutern, sondern die Inszenierung dieser Machtkommunikation aufzuzeigen, die über Verweisstrukturen (Zeichen, indirekte Reden), Raumstrukturen, Transgressionen (Geschenke, Botenblicke) funktioniert und im Blick auf das Buch in einer usurpierenden Erkenntnis gipfelt, über die das Fremde als falsches Eigenes klassiert wird. Dabei wird das Buch neben dem Bett des Alten vom Berge in der Darstellung Joinvilles zum Ort und Mittel einer exkludierenden Vereinnahmung des Fremden in den eigenen Wissenshorizont.

\section{Verweisstrukturen}

Körper und Zeichen im Raum (§ 451)

Die Inszenierung der Körper im Raum sowie die Kommunikation über Zeichen spielt von Anfang an eine entscheidende Rolle. So empfängt Ludwig in Akkon Gesandte des Alten vom Berge, die sich dabei so vor den König stellen, dass der Emir (l'amiral) hinter sich einen jungen Mann mit drei ineinandergesteckten Messern stehen hat, hinter dem ein dritter steht mit einem feinen Leintuch (un bouqueran) um den Arm gewickelt. Über die Erklärung Joinvilles werden die Messer und das Tuch (ein potentielles Leichentuch) als stille Todesdrohungen deutlich, da sie bei einem ungünstigen Verlauf des Empfangs zum Zug

9 Vgl. dazu Smith (Anm. 7), S. 62f.; Christine Ferlampin-ACher: Joinville, de l'hagiographe à l'autobiographe: approche de La Vie de saint Louis. In: Jean de Joinville. De la Champagne aux royaumes d'outre-mer. Hrsg. von D. QUÉREL, Saints-Geosmes 1998, S. 73-91; STARK (Anm. 7).

10 Zur Zeit Ludwigs IX war dies Tā $\square$ ad-Dīn, der seinen Sitz in Masyāe, in den syrischen Ber-gen hatte. Vgl. LEWIS (Anm.1), S. 120. 
gekommen wären. Auffallend ist, dass Joinville über seine Darstellung die klar als Drohung zu lesende Aufstellung der Gesandten vor dem König als Anordnung des Königs zeigt: Quant le roy revint de sa messe, il les fist venir devant li. Le roy les fist asseoir en tel maniere que ... (§ 451; „Als der König aus seiner Messe kam, ließ er sie vor sich kommen. Der König ließ sie sich in der Weise setzen, dass ..."). Über die Art der Formulierung wird selbst die Drohchoreographie der Gesandten auf die Macht des französischen Königs zurückgeführt.

Macht der vermittelten Worte: Gerücht und indirekte Rede (§ 452)

Erst nachdem die signifikante Anordnung der Gesandten im Raum und ihre zeichenhaften Drohgebärden in einem eigenen Abschnitt vorgestellt sind, berichtet Joinville von einer verbalen Auseinandersetzung. Auf die Frage des Königs, was sie von ihm wollten, antwortet der Gesandte: „Mein Herr hat mich geschickt, um zu wissen, ob Ihr ihn kennt“ (Mes sire me envoie a vous demander se vous le cognoissiés; § 452). Die Antwort des Königs, dass er ihn kaum kenne, da er ihn nie gesehen, nur von ihm gehört hätte, differenziert zwischen der Kenntnis durch Hörensagen und dem Augenzeugen-Wissen, wodurch das erstere entschärft wird. Aber genau darauf, auf dem Angst erzeugenden Ruf, gründet sich die Macht des Alten vom Berge, was die Nachfrage des Gesandten, warum er seinem Herrn dann nicht auch Tribut zolle wie andere Herrscher, die genau gewusst hätten, dass sie nur so lange leben, wie es dem Meister gefällt, deutlich macht. ${ }^{11}$

Auch hier ist Joinville bemüht, über die Darstellung die Macht des Königs gegenüber den Gesandten des Alten vom Berge als intakte zu zeigen. So erscheint die Anfangsfrage des Königs als Befehl zu reden: Le roy dit a l'amiral que il li deist sa volenté (§ 452; „Der König sagte dem Emir, dass er ihm sein Begehr sagen solle“). Und indem in der ganzen Episode kein direktes Wort des Königs berichtet wird, wird dieser im Text in eine ähnlich vermittelte Präsenz gerückt wie der nur über die Boten und die Gerüchte präsente Alte vom Berge.

Die über die Bedrohung aufgebaute Machtordnung wird jedoch nicht nur durch die Darstellung Joinvilles unterlaufen, sondern auch durch die im nächsten Paragraphen von den Gesandten vorgebrachte Handlungsalternative für den König irritiert: Falls der König es vorzieht, kann er auch anstelle von eigenen Zahlungen darauf hinwirken, dass der Alte vom Berge den Templern und Hospitalitern keinen Tribut mehr zahlen muss. ${ }^{12}$ Dies verschiebt die Hierarchie

11 Vgl. zu den Verhandlungen von Friedrich II. mit den Assassinen sowie den Tributforderungen und -einholungen durch die Hospitaliter und Templer DAFTARY (Anm. 1), S. 420f.

12 Die Assassinen waren mindestens seit 1228 den Hospitalitern tributpflichtig. Vgl. dazu DAFTARY (Anm. 1), S. 420f. Zu früheren Tributverhandlungen mit den Templern vgl. JEAN RICHARD: Vassaux, tributaires ou alliés? Les chefferies montagnardes et les Ismaîliens dans l'orbite des Etats des Croisés. In: Die Kreuzfahrerstaaten als multikulturelle 
zugunsten des Königs, dem Einfluss auf die Orden zugestanden wird. Ein Einschub Joinvilles erklärt diese Macht der Orden gegenüber den Assassinen strukturell, indem die institutionalisierte Hierarchie, in der jeder ersetzbar ist, nicht geschwächt werden kann durch die Ermordung eines einzelnen Machtträgers. Diese kleine, struktursoziologische Reflexion Joinvilles dient an dieser Stelle auch dazu, die charismatische Macht des Königs zu schützen, der sich im Folgenden auf die Macht der Orden stützen muss. ${ }^{13}$ Der König verspricht Antwort am Nachmittag und erwirkt sich dadurch den kleinen zeitlichen Aufschub, der ihm eine entsprechende Inszenierung seiner Machtposition erlaubt.

\section{Raumstrukturen}

Sprach- und Machträume (§§ 454-455)

Am Nachmittag empfängt der König die Gesandten, flankiert von den zwei Großmeistern der Orden. Der über das Gerücht konstituierten Macht einer verdeckt agierenden, streng hierarchischen Organisation mit einem charismatischen Führer wird die sichtbare Präsenz einer Machtvernetzung entgegengehalten, in der sich der König als durch institutionelle Strukturen gestützter charismatischer Kern inszeniert. In dieser neuen Situation weigern sich die Gesandten ihre Forderungen zu wiederholen, müssen dann aber dem Befehl der Ordensmeister gehorchen. Auch hier nimmt der Bericht von Joinville durch die Differenzierung von direkter und indirekter Rede eine subtile Hierarchisierung der intrikaten Machtverhältnisse zugunsten des Königs vor: während dieser wieder nur indirekt zu Wort kommt (Lors li dit le roy que il li redeist ce que il li avoit dit au matin, § 454; „,Der König sagte ihm, dass er wiederholen solle, was er ihm am Morgen gesagt hatte"), äußern sich die Ordensmeister in direkter Rede (Nous vous commandons que vous le dites, § 454; „Wir befehlen Euch es zu sagen"). Dadurch wird auf der textuellen Ebene eine klare Trennung eingeführt zwischen der Kommunikationsebene, auf der sich die Ordensgroßmeister mit den Gesandten direkt verbal auseinandersetzen und der indirekten Teilhabe daran durch den König und den Alten vom Berge. Noch deutlicher wird diese Differenzierung, wenn die darauf folgende Auseinandersetzung zwischen den Ordensmeistern und den Gesandten auf Arabisch stattfindet (en sarrazinois). In diesem fremden Sprachraum ist sie dem Bereich des Königs ganz entzogen.

Gesellschaft. Hrsg. von HANS EBERHARD MAYER, München 1997, S. 141-152, hier: S. $150 f$.

13 Zu charismatischer und institutioneller Macht vgl. MAX WEBER: Die charismatische Macht und ihre Umbildung. In: Ders.: Wirtschaft und Gesellschaft. Grundriss der verstehenden Soziologie. 5. rev. Aufl. besorgt von JoHANnES WincKeLMANN, Tübingen 1972, S. $654-$ 687. 
Und schließlich wird diese Loslösung der scharfen und harten Verhandlung vom König auch noch räumlich umgesetzt, indem den Gesandten befohlen wird, sich am nächsten Tag im Hospital einzustellen. Da werden sie dann nicht nur massiv bedroht und für ihre Unverfrorenheit dem König gegenüber abgestraft, sondern es wird ihnen befohlen, zur Wiedergutmachung in 14 Tagen mit Geschenken ihres Herrn für den König zurückzukommen (§ 455).

\section{Transgressionen}

Machtgeschenke: zeichenhafte Repräsentation und usurpierende Präsenz (§§ 456-458)

Die auf diese erste Begegnung folgende Kommunikation mittels Gesandtschaften zwischen dem Alten vom Berge und dem König ist initiiert und damit auch weitgehend gesteuert durch die Ritterorden. Denn tatsächlich bringen vierzehn Tage später die Gesandten des Alten vom Berge Briefe und Geschenke, die einerseits laut mitgelieferter Erklärung Zeichen der engen Verbundenheit des Absenders mit dem König sind (das Hemd des Alten vom Berge, ein Ring mit seinem Namen), anderseits als exquisitest gearbeitete Kleinode den Reichtum und die Macht des Absenders bezeugen (Elefanten, Giraffen und Äpfel aus Kristall, umwunden von goldenen Ranken mit Ambrablumen, Brett- und Schachspiele). Sowohl das Hemdgeschenk wie der Ring, der als Ehering erklärt wird, imaginieren in ihrer Auslegung den König in eine physische Nähe zum Alten vom Berge, die den König in eine Verbundenheit zwingt, die genauso Freundschaftsbeweis wie Vereinnahmung ist. Und durch den raumfüllenden Wohlgeruch, der der Geschenkkiste entströmt, ist der Absender auch plötzlich omnipräsent: et sachiez que si tost comme les messages ouvrirent leur escrins la ou ces choses estoient, il sembla que toute la chambre feust embausmee, si souef fleroient ( $\$ 457$; ,Und wisst, dass es, sobald die Boten ihre Schreine öffneten in denen diese Dinge waren, schien, als wenn der ganze Raum duften würde, so gut war ihr Geruch“). Die kostbaren Geschenke zur Ehrung des Empfängers sind immer auch Indizien der Macht des Absenders. Und die Geschenke des Alten vom Berge unterwandern die von den Ordensmeistern inszenierte Hierarchie durch die über Ring und Hemd initiierte Imagination der physischen Nähe sowie über den sich in den hintersten Winkel des königlichen Raums ausbreitenden fremden Duft.

Wenn der König dann als Antwort auch Geschenke zurückschickt, die jedoch, was auch die zurückhaltende Beschreibung Joinvilles unterstreicht, die erhaltenen Preziosen höchstens quantitativ (grant foison, eine grosse Menge), aber nicht qualitativ übertreffen können, lässt er diese Gesandtschaft begleiten durch den arabischsprechenden Dominikaner Yves le Breton. Mit dessen Blick 
und dessen Ohr dringt man nun im Bericht von Joinville in den Bereich des Alten vom Berge ein - bis ins Schlafzimmer. Damit wird in der Darstellung Joinvilles der ins Innerste eindringende Blick des Mönchs zu einer Art Widerpart zu dem alles durchdringenden Duft der Preziosen. Und die in den Geschenken des Alten vom Berge imaginierte Nähe als Vereinnahmung wird beantwortet durch eine investigierende Annäherung.

\section{Einblicke - tradiertes Wissen und Entdeckung $(\S \S 459-462)$}

Yves le Breton entdeckt, dass le Viel de la Montaingne nicht an Mohammed glaubt, sondern an la loi de Haali, das Gesetz von Ali. Dieser, als Onkel von Mohammed, hätte, laut dem bei Joinville wiedergegebenen Wissen, Mohammed zur Macht verholfen, dann aber, von diesem fallengelassen, eine eigene Anhängerschaft aufgebaut, die sich in Opposition zur Lehre von Mohammed konstituierte. Auch wenn diese Herleitung der Spaltung des Glaubens schon nur genealogisch nicht korrekt ist, ${ }^{14}$ stellt Joinville die Assassinen in Opposition zum orthodoxen (sunnitischen) Islam, dessen Begründer gleichzeitig als machthungrig und treulos charakterisiert wird:

Dont encore il est ainsi que touz ceulz qui croient en la loy Haali dient que ceulz qui croient en la loy Mahommet sont mescreant, et aussi touz ceulz qui croient en la loy Mahommet dient que touz ceulz qui croient en la loy Haali sont mescreant. (§ 459)

In der Folge davon ist es so, dass alle, die an das Gesetz von Ali glauben sagen, dass die, die an das Gesetz von Mohammed glauben ungläubig sind, und diejenigen, die an das Gesetz von Mohammed glauben sagen, dass alle, die an das Gesetz von Ali glauben ungläubig sind.

Zwei Punkte des Gesetzes von Ali (loy Haali), auf das sich die Assassinen beziehen, werden genannt: die Überzeugung, dass der Tod im Dienst des Meisters eine Wiedergeburt in einem besseren Körper bringe $(\S 460)^{15}$, und der Glaube an eine absolute Prädestination, was Joinville deutlich zurückweist: et ce ne doit nulz croire ( $\$ 461$; ,,aber dies soll niemand glauben“).

Während dieser kleine Exkurs zum Glauben der Assassinen Wissen referiert ohne Referenz auf Yves, nimmt die Darstellung mit dem Blick von Yves le

14 Die historisch nicht korrekte Darstellung vom Verhältnis von Mohammed und Ali, der dessen Schwiegersohn und nicht Onkel war, gibt eine Art Ursprungsgeschichte der Trennung von Sunna und Šī‘a. Einer der Ersten, wenn nicht der Erste, der die Spaltung im islamischen Glauben in seine Darstellung aufnahm war Wilhelm von Tyrus. Vgl. dazu BERNHARD HAMILTON: Knowing the Enemy. Western Understanding of Islam at the Time of the Crusades. In: Journal of the Royal Asiatic Society 7 (1997), S. 373-387, hier: S. 376.

$15 \mathrm{Zu}$ der Vorstellung von Seelenwanderung bei den Assassinen vgl. DAFTARY (Anm. 1), S. 401, wo u.a. auf nusairische Einflüsse hingewiesen wird; HEINZ HALM: Die islamische Gnosis, Zürich, München 1982, S. 291; RudOLF STROTHMANN: Seelenwanderung bei den Nusairī. In: Oriens 12 (1959), S. 89-114. 
Breton in den innersten Bereich der assassinischen Machträume wieder das Narrativ des Entdeckens auf:

Frere Yves trouva un livre au chevés du lit au Vieil, la ou il avoit escript pluseurs paroles que Nostre Seigneuer dit a saint Pere quant il aloit par terre. Et frere Yves li dit: „Ha, pour Dieu, sire, lisiés souvent ce livre, car ce sont trop bones paroles“. Et il dit que si fesoit il, „car j'ai moult chier mon seigneur saint Pere, car en l'encommencement du monde, l'ame de Abel, quant il fut tué, vint ou cors de Noé, et quant Noé fu mort, si revint ou cors de Habraham; et du cors Habraham, quant il morut, vint ou cors saint Pierre quant Dieu vint en terre“. (§ 462)

Bruder Yves fand ein Buch am Kopfende des Bettes des Alten, wo verschiedene Worte unseres Herrn aufgeschrieben waren, die er dem Hl. Petrus sagte, als er auf Erden wandelte. Und Bruder Yves sagte zu ihm: „Ha, bei Gott, Herr, lest dieses Buch oft, denn es sind sehr gute Reden.“ Und er sagte, dass er das tue, „,enn ich habe unseren Herrn Sankt Peter sehr gern, denn am Anfang der Welt kam die Seele Abels, als er getötet wurde, in den Körper von Noah, und als Noah starb, kam sie wieder im Körper von Abraham; und vom Körper Abrahams, als dieser starb, kam sie in den Körper von Petrus, als Gott auf die Welt kam.

In der Raumsemantik mittelalterlicher Architektur ist das Schlafgemach der innerste, intimste und geheimnisvollste Bezirk eines Gebäudes. ${ }^{16}$ Wenn Bruder Yves nun hier, in diesem intimsten aller Räume des Machtbereichs des Alten vom Berge, ein Buch findet, wird diese Entdeckung zum zentralen Moment des Eindringens in den fremden Glaubens- und Wissensraum. Indem dieser intime Blick - anders als das vorher referierte Wissen über den Glauben der Assassinen - in ein Narrativ direkten Erlebens gebunden ist, wird er auch zum Instrument einer Transgression in den an sich verschlossenen Bezirk unzugänglichen Wissens. Bei Joinville ist der Blick auf das Buch Endpunkt einer Darstellung des Glaubens der Assassinen, die mit der Abgrenzung von Mohammed beginnt, die Vorstellung von Reinkarnationsstufen nennt und die Lehre einer strengen Prädestination erwähnt. Es ist aber auch der Punkt, an dem der Blick des Mönchs in dem ganz Fremden plötzlich ein Eigenes sieht: Das im Kern des Unvertrauten und enigmatisch Fremden entdeckte Buch, im Herz der Finsternis sozusagen, öffnet sich auf einen vertrauten Textraum hin: Sprüche Christi an Petrus. Somit wird über den im Text nachvollzogenen Blick von Yves le Breton auf das Buch ein Geheimnis gelüftet und darüber ein fremder Wissensraum aufgebrochen; über den bei Joinville inszenierten Blick vollzieht sich Entdeckung und Verrat in einem.

16 Vgl. dazu KARIN LERCHNER: Lectulus floridus. Zur Bedeutung des Bettes in Literatur und Handschriftenillustration des Mittelalters, Köln 1993 (Pictura et poesis 6); MIREILLE SCHNYDER: Topographie des Schweigens. Untersuchungen zum deutschen höfischen Roman um 1200, Göttingen 2003 (Historische Semantik 3), S. 336ff. 


\section{Aneignung und Entfremdung}

\section{Schrift und Wort (§§ 462-463)}

An die Entdeckung des Buches schließt sich ein durch Joinville als direkte Rede wiedergegebenes Gespräch zwischen Bruder Yves und dem Alten vom Berge an. Dass es sich bei den im Buch aufgeschriebenen Reden Christi an Petrus um trop bones paroles, sehr gute Sprüche, handelt, sind sich die zwei einig. Die Differenz ergibt sich dann in der Begründung dieser Wertung und der Einordnung der Protagonisten in ein Heilssystem: Petrus, für Yves ein Apostel, ist dem Alten vom Berge wichtig, weil er eine Inkarnation der Seele Abels ist. ${ }^{17}$ Die Übereinstimmung im Blick auf den schriftlichen Text wird durch die mündliche Exegese zerstört. Damit öffnet sich die Szene - im Gespräch über Petrus plötzlich auf die Problematik von christlicher Orthodoxie und Häresie, von richtiger und falscher Exegese, von Schriftwahrheit und Auslegungswahrheit. Die Assassinen, eingeführt als Anhänger einer islamischen Gegenpartei zu Mohammed, werden so unter der Hand zu christlichen Häretikern.

Die hier inszenierte Entdeckung von Worten Christi durch Bruder Yves im Kern des fremden Glaubensgebäudes mag als ein Widerhall kursierender Gerüchte gesehen werden, nach denen die Assassinen zum Christentum konvertierten. ${ }^{18}$ Das Kopfkissenbuch des Alten vom Berge wäre so eine durch religions- und machtpolitische Hoffnungen ausgelöste Projektion.

Schon in anderem Zusammenhang hat sich aber Bruder Yves in eine Episode hineinerzählt, die er nicht erlebt, sondern höchstens gehört oder gelesen haben kann. ${ }^{19}$ So kann der Blick auf die Bettlektüre des Alten Mannes vom Berge auch ein narratives Arrangement von Bruder Yves sein, der offensichtlich

17 In der komplexen, zyklischen Geschichts-Struktur von sich in der Zeit perfektionierenden Perioden, die jeweils durch einen Propheten eingeleitet werden, der durch eine Vermittlerfigur begleitet ist, eine Art Exeget des Sinns, findet sich Petrus als Begleiter Christi. Die Reihe der Propheten beginnt mit Adam (begleitet von Abel) und endet mit Mohammed (begleitet von Ali). Dazu Halm (Anm. 15), S. 302; DAFTARY (Anm. 1), S. 139f.; W. MOntgomery WATt, Michael Marmura: Der Islam II. Politische Entwicklungen und theologische Konzepte, Stuttgart etc. 1985 (Die Religionen der Menschheit 25,2), S.456f.

18 Vgl. oben, Anm. 5.

19 In $\S 445$ erzählt Bruder Yves von einer zufälligen Begegnung auf dem Markt mit einer alten Frau, die Feuer und Wasser in den Händen hielt, das eine, um damit das Paradies zu verbrennen, das andere, um die Hölle zu löschen. Denn man sollte nicht aus Hoffnung aufs Paradies oder aus Furcht vor der Hölle Gutes tun, sondern allein um der Liebe Gottes willen. Es handelt sich dabei um eine von der 801 in Basra gestorbenen Mystikerin Rābi‘a al-'Adawīya überlieferte Geschichte. Vgl. zu dieser Geschichte: MARIE-THERESE D'AlVERNY: La connaissance de l'Islam au temps de Saint Louis. In: Septième centenaire de la mort de saint Louis, Actes des colloques de Royaumont et de Paris, 21-27 mai 1970. Paris: CNRS et Société d'Edition „Les Belles Lettres”, 1976, S. 235-246, hier: S. 238. [wieder abgedruckt in: Dies..: La connaissance de l'Islam dans l'Occident médiéval. Hrsg. von CH. BURNETT, Vermont 1994, Kap. 6]. 
aus eigener Lektüre oder vom Hörensagen einige Kenntnis von arabischislamischer Tradition hat und sich entsprechend als intimer Kenner des fremden Glaubens in Szene setzt. Der Blick auf das Kopfkissenbuch des Alten vom Berge wäre dann eine aktualisierende und authentisierende Selbstinszenierung im Rahmen einer Vermittlung andernorts erworbenen Wissens. Dazu können auch gewisse Kenntnisse von Glaubensinhalten der nizāritischen 'Ismā'īliten (der Assassinen) gehören, in deren stark gnostisch beeinflusstem Glaubenssystem Petrus einen festen Platz hatte. ${ }^{20}$

Die Bettlektüre des Alten vom Berge bei Joinville kann so durchaus auch im Rahmen des nizāritischen Glaubenssystems plausibilisiert werden. Unter den frühen apokryphen Petrus-Schriften finden sich Texte, die 'ismā‘īlitischen Glaubensvorstellungen nahe kommen, wie z.B. die in Syrien in gnostischem Umfeld entstandenen Kerygmata Petrou. ${ }^{21}$ In ihnen ist unter anderem auch die Idee des ,wahren Propheten“ vertreten, der sich seit Anfang der Welt in einer fortlaufenden Reihe von Gestalten manifestiert, wobei Adam seine erste Inkarnation ist. Es ist insofern nicht unwahrscheinlich, dass ein Buch ähnlicher Art sich bei den Assassinen fand.

Und doch bleibt in dem Bericht von Joinville eine Irritation. Denn in der Reihe der Inkarnationen von Abels Seele, wie sie Bruder Yves in seinem Bericht dem Alten vom Berge in den Mund legt, erscheinen ausschließlich Figuren der jüdisch-christlichen Tradition: Abel, Noah, Abraham, Petrus - die muslimischen Figuren dieser Inkarnationsreihe sind weggelassen. Darüber wird sie aus dem islamischen Zusammenhang gelöst. Im von Joinville wiedergegebenen Blick von Bruder Yves wird so tradiertes Wissen über den fremden Glauben von christlicher Wunschprojektion überlagert, die die Assassinen als konvertierte Christen sehen will. ${ }^{22}$

20 Es ist vor allem die von den Assassinen verbreitete ,neue Lehre', die sich stark an die frühere Gnosis anbindet. Vgl. dazu HALM (Anm. 15), S. 8-16; HENRY CORBIN: From the Gnosis of Antiquity to Ismaili Gnosis. In: Ders.: Cyclical Time and Ismaili Gnosis. London u.a. 1983, S. 151-193. Zu der 'ismā‘īlitischen Lehre siehe auch: DAFTARY (Anm. 1). Zu Petrus vgl. oben, Anm. 17.

21 G. STRECKER: Die Kerygmata Petrou. In: EDGAR HENNECKE: Neutestamentliche Apokryphen in deutscher Übersetzung. 3., völlig neubearb. Aufl. hrsg. von WILHELM SCHNEEMELCHER, Bd. II, Tübingen 1964, S.63-80. Vgl. zu Entstehungsort und gnostischem Umfeld ebd. S. 63-69. Auch BERNHARD HAMILTON kommt zu der Vermutung, dass das bei Joinville erwähnte Buch mit diesen Texten in Verbindung zu bringen sein könnte: HAMILTON (Anm. 14), S. 377, Anm. 18. Zu einem in 'ismā'īlitischer Literatur erwähnten Petrus-Buch (kitāb Butrus) vgl. PAUL KRAUS: Hebräische und syrische Zitate in 'ismā'īlitischen Schriften. In: Ders.: Alchemie, Ketzerei, Apokryphen. Gesammelte Aufsätze. Hrsg. und eingeleitet von RÉMI BRAGUE, Hildesheim, Zürich, New York 1994, S. 1-23, hier S. 12.

22 Die Interpretationen, dass diese Stelle ein Hinweis auf die Toleranz Joinvilles sei, der den Assassinen (Muslimen) sogar die Inkarnation zuschreibe, ist auf diesem Hintergrund nicht nachvollziehbar. Vgl. YVETtE GuILChER-PELlat: Joinville en paennime, l'autre, 1'ailleurs. In: QuEREL (Anm. 9), S. 193-206, hier: S. 205. 
Der in Joinvilles Bericht zuerst als muslimischer Häretiker gezeigte Alte vom Berge wird im Blick auf sein Kopfkissenbuch zum Christen, durch das Gespräch über den Text dann aber zum christlichen Häretiker. Das ungelesene, nicht ausgelegte Buch stiftet eine vermeintliche Gemeinschaft, die im Akt der sinnstiftenden Lektüre, der Exegese, sich auflöst. Ist das geschriebene Buch vertraut, ist das ausgelegte Buch fremd. Die sich im gesprochenen Wort aufreißende Differenz ist aber nicht zu überwinden: Alle Bemühungen von Bruder Yves, dem Alten vom Berge seine Irrlehre vor Augen zu halten und ihn eines Besseren zu belehren, fruchteten nichts: mes il ne le volt croire $(\S 463$; ,aber er wollte es nicht glauben“). 
Bereitgestellt von | UZH Hauptbibliothek / ZB Zuerich (UZH Hauptbibliothek / ZB Zue Angemeldet | 172.16.1.226

Heruntergeladen am | 22.03.12 11:10 